\title{
Evolution of microstructure and variations in mechanical properties accompanied with diffusionless isothermal $\omega$ transformation in $\beta$-titanium alloys
}

\author{
Norihiko L. Okamoto $\odot,{ }^{1,}{ }^{*}$ Shuhei Kasatani, ${ }^{1}$ Martin Luckabauer $\odot,{ }^{1,2}$ Robert Enzinger $\odot,{ }^{3}$ Satoshi Tsutsui $\odot,{ }^{4,1,5}$ \\ Masakazu Tane $\odot,{ }^{6,7}$ and Tetsu Ichitsubo $\odot^{1, \dagger}$ \\ ${ }^{1}$ Institute for Materials Research, Tohoku University, Sendai, Miyagi 980-8577, Japan \\ ${ }^{2}$ Faculty of Engineering Technology, University of Twente, P.O. Box 217, 7500AE Enschede, The Netherlands \\ ${ }^{3}$ Institute of Materials Physics, Graz University of Technology, Graz, 8010, Austria \\ ${ }^{4}$ Japan Synchrotron Radiation Research Institute (JASRI), Sayo, Hyogo 679-5198, Japan \\ ${ }^{5}$ Institute of Quantum Beam Science, Graduate School of Science and Engineering, Ibaraki University, Hitachi, Ibaraki 316-8511, Japan \\ ${ }^{6}$ The Institute of Scientific and Industrial Research, Osaka University, Osaka 567-0047, Japan \\ ${ }^{7}$ JST, PRESTO, 4-1-8 Honcho, Kawaguchi, Saitama, 332-0012, Japan
}

(Received 14 September 2020; accepted 17 November 2020; published 9 December 2020)

\begin{abstract}
Diffusionless isothermal omega (DI- $\omega$ ) transformation, which was recently defined in Phys. Rev. Materials 3, 043604 (2019), is classified into a third category of the $\omega$ transformation modes, other than the well-known isothermal and athermal modes. This work reveals the characteristic features of the DI- $\omega$ transformation in the $\beta$-titanium vanadium alloy system, specifically, focusing on variations in the microstructure and mechanical properties with the proceeding of the DI- $\omega$ transformation. After quenching alloys of Ti-15at $\% \mathrm{~V}$, Ti-21at $\% \mathrm{~V}$, and $\mathrm{Ti}-27 \mathrm{at} \% \mathrm{~V}$ down to below room temperature from the $\beta$-stable temperature, in addition to the occurrence of the athermal $\omega$ transformation for Ti- $15 \mathrm{at} \% \mathrm{~V}$, all of the alloys gradually undergo the DI- $\omega$ transformation upon aging at a temperature as low as $373 \mathrm{~K}$, leading to a moderate increase in the hardness. The degree of the hardness increase in these alloys can be successfully explained by a local instability concept based on quenched-in thermal concentration fluctuations. It is also shown that internal friction (Ti-21at\%V) diminishes after the low-temperature aging, which indicates the annihilation of such unstable regions showing a dynamic collapse of $\{111\}_{\beta}$ pairs to form a transient DI- $\omega$ phase. Comparison of inelastic x-ray scattering and ultrasound measurements can see a trail of the DI- $\omega$ transformation remaining even in the Ti-27at\%V alloy, which has no obvious athermal omega transformation temperature. Based on the results, the difference between athermal $\omega$ and DI- $\omega$ transformations is finally discussed.
\end{abstract}

DOI: 10.1103/PhysRevMaterials.4.123603

\section{INTRODUCTION}

Titanium alloys have been indispensable materials for modern industrial society in that they exhibit superior properties such as mechanical strength, corrosion resistance, and biocompatibility. Specifically, $\beta$-Ti alloys have received great attention due to their excellent properties [1-7]. However, it is known that the hexagonal omega $(\omega)$ phase [8], which is brittle and usually undesired for mechanical applications, is unfortunately formed from the unstable $\beta$ phase quenched from high temperatures where the $\beta$ phase is thermodynamically stable. Therefore, to harness the superior properties of the $\beta$ phase, it is crucial to grasp the nature and control the kinetics of the $\omega$ transformation especially when $\beta$-Ti alloys are used in practical applications.

Many researchers reported significant works on the mechanisms of the $\omega$ transformation [9-19]. From a crystallographic point of view, the $\omega$ transformation in $\beta$-Ti alloys usually occurs via the collapse of $\{111\}_{\beta}$ plane pairs [10], which is

\footnotetext{
*nlokamoto@tohoku.ac.jp

†tichi@imr.tohoku.ac.jp
}

often interpreted as the dynamic response to a softening of the $2 / 3[111]_{\beta}$ longitudinal phonon mode, as shown in Fig. 1(a). This $\{111\}_{\beta}$ collapse looks very similar to the shuffling motion in premartensitic transformations [20-26]. From a thermodynamic point of view, it has been considered that the $\omega$ transformation process falls into either "athermal" or "isothermal" modes $[10,11]$. The athermal $\omega$ transformation takes place via a diffusionless mechanism at temperatures where atomic diffusion cannot occur, whereas the isothermal $\omega$ transformation proceeds through a diffusion-mediated phase separation at temperatures (above $\sim 500 \mathrm{~K}$ ) where atomic diffusion can take place appreciably [11]. Specifically, the first mode occurs instantaneously like a martensitic transformation, while the second mode exhibits an incubation time for the $\omega$ transformation because atomic diffusion is required for the solute-atom partitioning to form solute-lean regions [11].

Several researchers have, however, pointed out that there exists a peculiar transformation mode that cannot be classified into either of the two conventional modes [27-29]; a diffuse $\omega$ structure, which is an incomplete $\omega$ structure also termed "modulated omega" in the de Fontaine paper [10], can gradually be formed during isothermal aging at around room temperature (RT), where atomic diffusion is hardly expected 


\section{(a) Atomic-scale view}

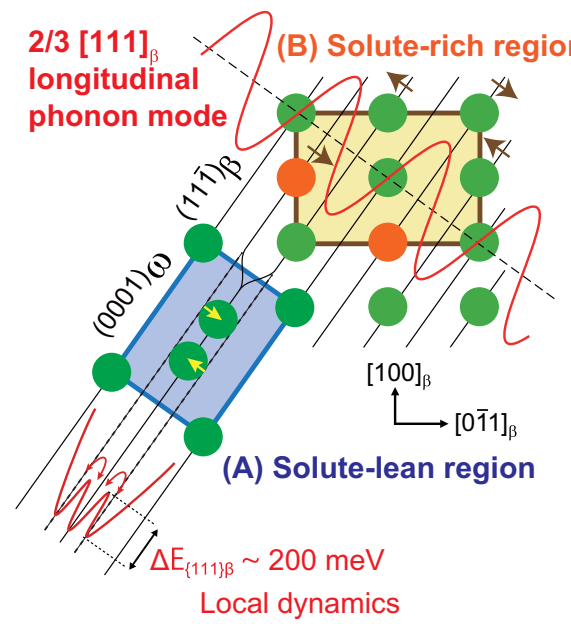

(b) Statistical view
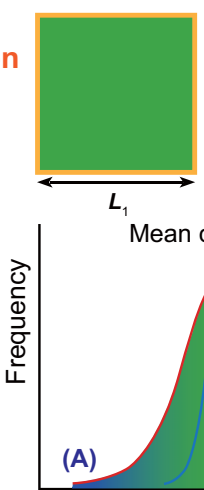

$L_{1}$

$\vec{L}_{2}$

Mean composition

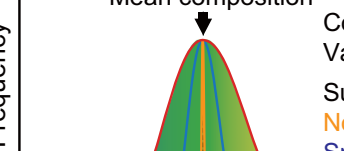

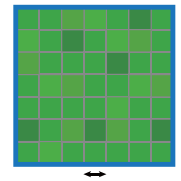

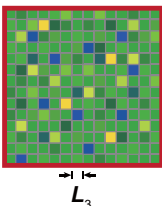

(c) Transformation mode

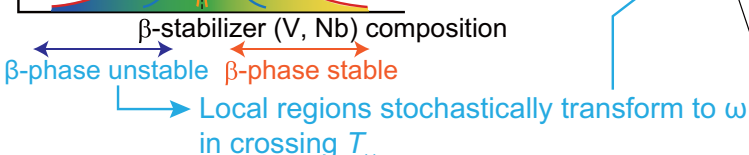

in crossing $T$

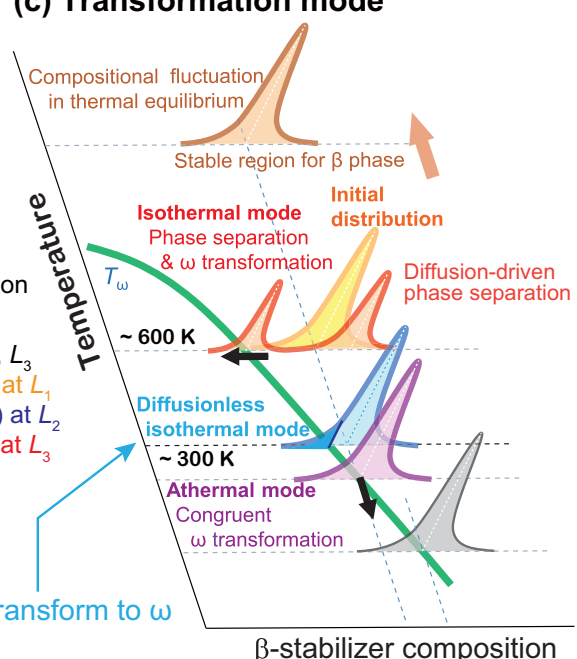

FIG. 1. Schematic illustration showing (a) the crystallographic relations of the $\beta \leftrightarrow \omega$ transformation with the $2 / 3\{111\}_{\beta}$ longitudinal wave, (b) the concentration fluctuation to be considered from the viewpoint of statistical mechanics whose magnitude is dependent on the size of subsystems in which the concentration is defined, and (c) the various modes in the $\omega$ transformation, where $T_{\omega}$ shown as the green line is the athermal $\omega$ transformation temperature as a function of the macroscopically averaged concentration of the sample, indicated by a yellow line in (b). The gray peak corresponds to a concentration distribution for a $\beta$-Ti alloy with a higher $\beta$-stabilizer concentration.

to occur in the $\beta$-Ti alloy systems. This phenomenon was also observed by one of the present authors [30]. It should be noted that the diffuse $\omega$ structure can be formed even if the aging temperature (RT) is much above the athermal $\omega$ transformation temperature $T_{\omega}$ [10]. and the athermal $\omega$ transformation does not finish in the entire $\beta$ matrix even when cooled down to $T_{\omega}$. In fact, the transformation only gradually proceeds upon further cooling below $T_{\omega}$ [10], although there is usually no large temperature hysteresis found for the $\beta \leftrightarrow \omega$ athermal transformation in systems where this transformation clearly occurs.

To clarify these unsolved problems and to construct a universal $\omega$ transformation theory, we recently explored the detailed mechanism of the diffusionless isothermal $\omega(\mathrm{DI}-\omega)$ transformation in the Ti-V alloy system [31]. Figures 1(b) and 1(c) summarize the fundamental mechanism of the $\omega$ transformation. In $\beta$-Ti alloyed with $\beta$-stabilizing elements such as $\mathrm{V}$ or $\mathrm{Nb}$, and so on, locally unstable regions poor in the $\beta$-stabilizing elements exist due to quenched-in compositional fluctuations. Note that this compositional fluctuation is not an inhomogeneity (failure) unexpectedly introduced during a sample fabrication process but the thermal equilibrium fluctuation that inevitably exists even in a single phase for a statistical reason. As described in the literature [31-33], the variance $\left(\sigma^{2}\right)$ of the concentration distribution is inversely proportional to the volume $L^{3}$ of each subsystem in which we define the local concentration, that is, $\sigma^{2} \sim k T / L^{3}$, where $k$ and $T$ are the Boltzmann constant and temperature, respectively. As shown in Fig. 1(b), since the quenched-in compositional fluctuation at the nanometer scale is large, some local regions are inevitably lean in the $\beta$-stabilizing element, and hence, become unstable for the $\beta$ structure. Therefore, the DI- $\omega$ transformation can locally occur even if the $\beta$-phase matrix seems macroscopically stable above $T_{\omega}$, as illustrated in the blue curve (blue region) of Fig. 1(c). In general, phase transitions are regarded as cooperative phe- nomena, and therefore many researchers tried to understand the $\omega$ transformation in this way. However, although the softening of the $2 / 3[111]_{\beta}$ longitudinal phonon mode plays an important role, our previous study [31] showed that this softening cannot be completely prohibited only by adding $\beta$-stabilizing elements ( $\mathrm{V}$ or $\mathrm{Nb}$, etc.). The $\omega$ transformation is not a conventional cooperative phenomenon and the local lattice instability [Fig. 1(a)] can inevitably take place in regions lean in $\beta$-stabilizing elements, and the DI- $\omega$ transformation can therefore proceed locally even above $T_{\omega}$, as indicated by the blue curve in Fig. 1(c).

In the present paper, we intend to clarify the relationship between mechanical properties and the DI- $\omega$ transformation that is a fluctuation-driven transformation and has not been previously proposed as a phase transformation mode in $\mathrm{Ti}$ alloys. Specifically, for $\beta$-Ti-V alloys, we more deeply investigate the characteristic features of the DI- $\omega$ transformation in terms of the microstructure evolution and mechanicalproperty change accompanying its progress. We show that these changes can be thoroughly interpreted by using the concept illustrated in Fig. 1, and characterized by means of differential scanning calorimetry, hardness measurements, transmission electron microscopy, submegahertz internalfriction measurements at low temperatures, and inelastic x-ray scattering measurements. What we emphasize here is that the various phenomena including room temperature aging can be successfully explained and understood based on the present mechanism.

\section{EXPERIMENT}

Ti-V alloy ingots were prepared from $99.9 \mathrm{wt} \%$ purity $\mathrm{Ti}$ and $99.7 \mathrm{wt} \%$ purity $\mathrm{V}$ by an arc-melting with tiltcasting apparatus. Three kinds of alloy ingots were prepared by inductively coupled plasma-optical emission spectrometry (ICP-OES, IRIS Advantage DUO, Thermo Fisher 
Scientific Inc., USA) and oxygen elemental analysis (TC436, LECO Corp., USA), the chemical compositions of the ingots were evaluated to be Ti-15.2at\%V (called Ti-15at $\% \mathrm{~V}$ ), $-21.2 \mathrm{at} \% \mathrm{~V}$ and $-20.9 \mathrm{at} \% \mathrm{~V}(\mathrm{Ti}-21 \mathrm{at} \% \mathrm{~V})$, and -26.9 at $\% \mathrm{~V}$ (Ti-27at\%V), and the oxygen concentration in the ingots was in the range of $0.7-1.2$ at $\%$. The effects of solute oxygen atoms are not the scope of the present study. For the interested reader a detailed report can be found elsewhere [34]; where we elucidated that solute oxygen atoms retard the DI- $\omega$ transformation. Based on previous works [31,35], the athermal $\omega$ transformation temperatures $T_{\omega}$ of the respective alloys are $T_{\omega}(15 \mathrm{~V}) \gg \mathrm{RT}$ for Ti-15at $\% \mathrm{~V}, T_{\omega}(21 \mathrm{~V})<\mathrm{RT}$ (or $T_{\omega}(21 \mathrm{~V})$ $\sim \mathrm{RT})$ for Ti-21at $\% \mathrm{~V}$, and $T_{\omega}(27 \mathrm{~V}) \ll \mathrm{RT}$ for Ti-27at $\% \mathrm{~V}$. All the samples were subjected to solid-solution treatment at $1073 \mathrm{~K}$ for $2 \mathrm{~h}$ in a vacuum atmosphere of $10^{-3} \mathrm{~Pa}$, followed by quenching in ice water. All the measurements were performed soon after (within $12 \mathrm{~h}$ ) quenching the samples to minimize unmonitored RT aging effects.

Prior to the investigation of the DI- $\omega$ transformation kinetics, we first monitored the kinetics of all the transformations of these alloys by differential scanning calorimetry (DSC). Additionally, a laser interferometric dilatometer was used to detect the isothermal sample expansion/contraction due to the DI- $\omega$ and isothermal $\omega$ transformation. After the solidsolution treatment, the samples were annealed at $373 \mathrm{~K}$ in air for various periods. To investigate the microstructure and hardness changes over time, transmission electron microscopy (TEM) observation (JEOL JEM-2000EX TEM operated at $200 \mathrm{kV}$ ) and Vickers hardness (VH) measurements (Shimadzu HMV-G21) were carried out. As for the TEM observation, thin foils were prepared before and after aging at $373 \mathrm{~K}$ for $16 \mathrm{~h}$. Foils with $3 \mathrm{~mm}$ in diameter and $200 \mu \mathrm{m}$ in thickness were machined from the heat-treated alloys, followed by mechanical grinding, polishing, and dimpling so that the thickness at the foil center becomes approximately $20 \mu \mathrm{m}$. Then, the foils were subjected to $\mathrm{Ar}^{+}$ion milling for perforation in a precision ion polishing system (PIPS II, Gatan, Inc.) operated at $4.0 \mathrm{kV}$ accelerating voltage, which was step-wise decreased to 2.0, 1.0, 0.5, 0.2, and $0.1 \mathrm{kV}$.

Furthermore, we investigated the aging (DI- $\omega$-formation) effects on the low-temperature internal friction $Q^{-1}$ in the Ti-21at\% $\mathrm{V}$ alloy by using electromagnetic acoustic resonance (EMAR) measurements. First, the quenched Ti-21at\%V alloy sample in a cylindrical shape was measured upon cooling from 300 to $4 \mathrm{~K}$, and subsequently, after annealing the same sample at $373 \mathrm{~K}$ for $72 \mathrm{~h}$, a similar cooling experiment was repeated. The resonance frequency $f_{\mathrm{r}}$ and internal friction $Q^{-1}$ were measured with the free-decay method; the detailed procedure of the EMAR technique is described in the literature [36].

In addition, we also determined the sound velocities (longitudinal elastic constants) along the $[111]_{\beta}$ axis by EMAR and inelastic $\mathrm{x}$-ray scattering (IXS) measurements to detect the relaxation phenomenon in the sample $(\mathrm{Ti}-27 \mathrm{at} \% \mathrm{~V})$ that exhibits no athermal $\omega$ transformation. Single crystals for the EMAR and IXS measurements were grown from the master alloys using a floating-zone method at a crystal growth rate of $2.5 \mathrm{~mm} \mathrm{~h}^{-1}$ in Ar atmosphere; the detailed procedure of preparing single crystals is described in our previous paper [31]. The IXS experiment was carried out at BL35XU of
SPring-8 [37]. A detector composed of $3 \times 4$ analyzer arrays was installed in the spectrometer of BL35XU. We chose the $\mathrm{Si}(111111)$ backscattering setup: the incident $\mathrm{x}$-ray energy was $21.747 \mathrm{keV}$ and the energy resolution was about $1.5 \mathrm{meV}$, slightly depending on the analyzer. In the present work, the $\vec{Q}$-resolution in the IXS measurements were $\Delta \vec{Q} \sim(0.010 .02$ 0.02) (r. l. u.). The single crystalline sample was put on the He-closed cycle cryostat installed in the IXS spectrometer at BL35XU. Rocking curve widths of ( $\left(\begin{array}{lll}2 & 2 & 2\end{array}\right)$ and $\left(\begin{array}{lll}1 & 2 & 3\end{array}\right)$ in the sample were less than 0.1 degree, which is smaller than the $\vec{Q}$-resolution of the IXS measurements.

\section{RESULTS AND DISCUSSION}

\section{A. Detection of DI- $\omega$ mode in differential scanning calorimetry and laser dilatometry}

Figure 2(a) shows the DSC curves obtained for samples with three different $\mathrm{V}$ compositions. By comparing the first and second scans, a small but obvious peak corresponding to DI- $\omega$ transformation can be observed around $300-500 \mathrm{~K}$ in the first heating scan for the quenched $\beta$-Ti alloys. It is interesting to note that the DI- $\omega$ transformation can take place even in the Ti-27at\% V alloy whose $T_{\omega}$ is much lower than RT or absent $[31,35]$. As shown later (in Fig. 3), since the Ti-15at\%V alloy shows an athermal $\omega$ transformation during quenching from $\beta$-phase solid-solution temperature, we have chosen the Ti-21at\%V alloy, which shows no obvious athermal $\omega$ transformation, for the isothermal contraction/dilatation measurement. Figure 2(b) shows the sample contraction obtained for the Ti-21at $\% \mathrm{~V}$ alloy with aging at various temperatures. The inset in Fig. 2(b) presents the diffusion distance as a function of annealing temperature, which is estimated by the previous diffusion coefficient data for Ti-19at $\% \mathrm{~V}$ by Mudock et al. [38]. Also based on this, we can judge that atomic diffusion in Ti-V alloys is impractical at temperatures below about $500 \mathrm{~K}$. Actually, as atomic diffusion can appreciably start to take place above $550 \mathrm{~K}$, substantial phase separation can be expected above this temperature, so that the conventional isothermal $\omega$ transformation occurs; this temperature region is very consistent with the previous work reported by Choudhuri et al. [14]. For all the annealing temperatures an isothermal contraction is found, which occurs with aging even at $373 \mathrm{~K}$. It should be noted that this does not necessarily mean that the atomic volume of the $\omega$ phase is smaller than that of the $\beta$ phase in the Ti-21at $\% \mathrm{~V}$ alloy. In fact, the negative sign of the length changes appearing may be understood as a result of the varying fractions of $\beta$-phase matrix that shrinks due to $\mathrm{V}$ enrichment and $\omega$-phase particles that have a very small positive eigenstrain [39], formed during a more or less strong decomposition of the alloy. The detailed analysis of the kinetics of the DI- $\omega$ and conventional isothermal $\omega$ transformations will be the topic of an upcoming publication.

Upon further heating in the DSC measurement, the inverse transformation from $\omega$ to $\beta$ (endothermic reaction), the transformation from $\beta$ to $\alpha$ (exothermic reaction), and one from $\alpha$ to $\beta$ phase (slight/broad endothermic reaction) are observed for all the samples; each peak assignment is based on previous work [6]. In the first cooling from $1050 \mathrm{~K}$, the Ti- 15 at $\% \mathrm{~V}$ alloy shows a segregation reaction of $\beta \rightarrow \alpha+\beta_{1}$, 

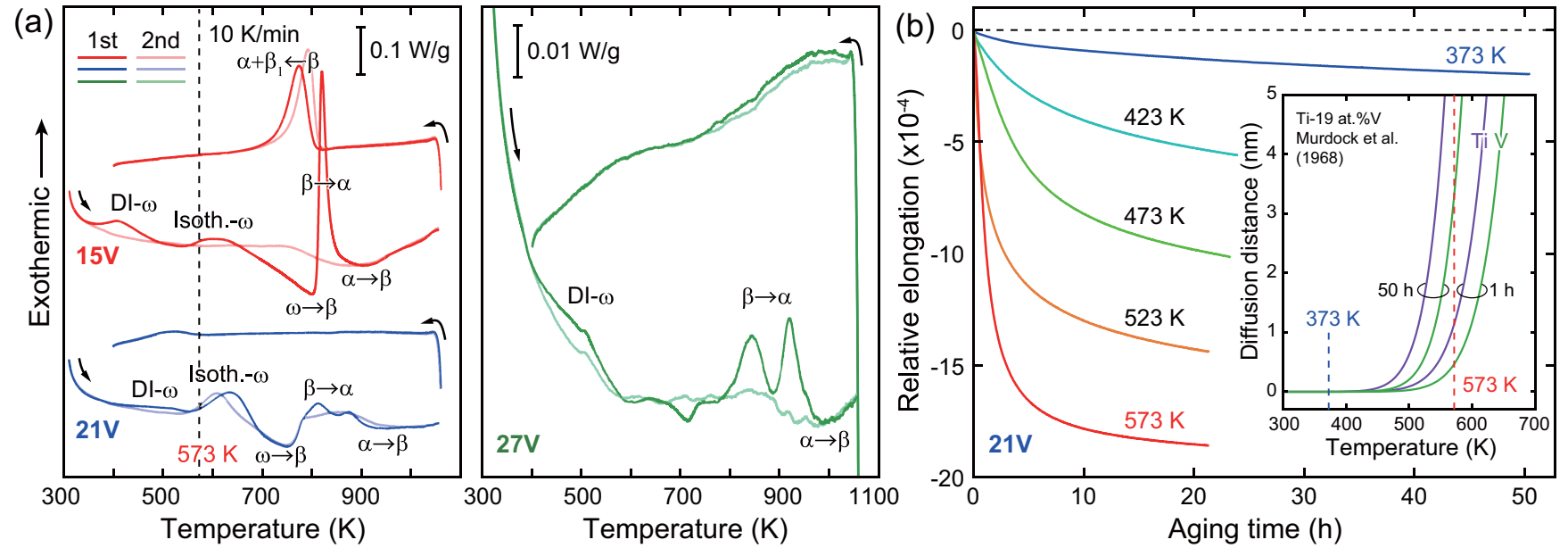

FIG. 2. (a) DSC measurements for Ti-15at $\% \mathrm{~V}$, Ti-21at $\% \mathrm{~V}$, and Ti-27at $\% \mathrm{~V}$ alloys and (b) laser dilatometry measurements for the Ti$21 \mathrm{at} \% \mathrm{~V}$ alloy; all the samples were quenched after the solid-solution treatment at $1073 \mathrm{~K}$. The inset in (b) shows the diffusion distance estimated by the tracer diffusion coefficients of $\mathrm{Ti}$ and $\mathrm{V}$ in Ti-19at $\% \mathrm{~V}$ alloys [38]. The diffusion distances are given by $\sqrt{2 D t}$ at each temperature for $t=1 \mathrm{~h}$ and $50 \mathrm{~h}$.

whereas the Ti-21at\% V and Ti-27at\%V alloys show no such segregation reaction at the cooling rate as fast as $10 \mathrm{~K} / \mathrm{min}$, which suggests that sufficient atomic diffusion is required for the $\alpha$ phase formation. Moreover, in the second heating scan, the DI- $\omega$ transformation cannot be observed for all the samples because the $\omega$ phase has already been formed during the cooling at the rate of $10 \mathrm{~K} / \mathrm{min}$ in the DSC analysis. In terms of this, it is suggested that the DI- $\omega$ transformation occurs only in the $\beta$ phase in the as-quenched state.

\section{B. Microstructural changes before and after aging at $373 \mathrm{~K}$}

In the above section, the DSC and laser dilatometry analyses have shown that the DI- $\omega$ transformation can appreciably proceed around $300-400 \mathrm{~K}$. Then, we also investigated the changes in microstructure and hardness with aging at $373 \mathrm{~K}$; this temperature is much lower than $550 \mathrm{~K}$, above which atomic diffusion occurs appreciably. Figures 3(a) to 3(f) show the results of the TEM observation for the asquenched (after solution treatment) and aged samples (at $373 \mathrm{~K}$ for $16 \mathrm{~h}$ ) of each composition; upper and lower pictures for each sample are a set of dark-field images and the electron diffraction patterns taken along the $[1 \overline{1} 0]_{\beta}$ direction, where the diffraction spot indicated by the yellow circle was used for the dark-field imaging. For reference, Fig. 3(g) schematically illustrates the reciprocal lattices of the $\beta+\omega$ phases when projected along the $[1 \overline{1} 0]_{\beta}$ direction; the diffraction spots that are absent in Fig. 3(g) are due to the multiple diffraction of electrons, and green and red spots represent the two kinds of the $\omega$ variants.

As is seen in Fig. 3(a), the $\omega$ phase is already formed after quenching the solution-treated Ti-15at\%V alloy, which means that the athermal $\omega$ transformation occurs during quenching and, therefore, $T_{\omega}$ of Ti-15at $\% \mathrm{~V}$ is clearly higher than RT. Comparing Figs. 3(a) and 3(d), despite marginal changes can be observed in electron diffraction patterns after aging at $373 \mathrm{~K}$ for $16 \mathrm{~h}$, the proportion of bright areas in the dark-field image slightly increases due to aging, indicating the diffuse $\omega$ phase was formed by aging. In contrast, the diffraction spots attributed to the $\omega$ phase can be hardly seen in the electron diffraction pattern of the quenched Ti-21at\% V alloy [Fig. 3(b)], and accordingly, the dark-field image is substantially homogeneous in the as-quenched state, although faint diffuse scattering is observed near the diffraction spots of the $\omega$ phase in addition to the diffraction spots corresponding to the $\beta$ phase. However, as seen in Fig. 3(e), the diffraction spots from the $\omega$ phase appear in the electron diffraction pattern after aging at $373 \mathrm{~K}$ for $16 \mathrm{~h}$, and the corresponding dark-field image confirms that the nanometer-scale $\omega$ phase is formed during the aging. These results suggest that the quenched Ti-21at $\% \mathrm{~V}$ alloy consists mainly of the $\beta$ phase (but includes extremely fine diffuse $\omega$ phase) and $T_{\omega}$ of the Ti-21at $\% \mathrm{~V}$ alloy is near or slightly higher than RT. Consequently, we judged that the DI- $\omega$ transformation can proceed at $373 \mathrm{~K}$, where diffusion can hardly occur.

As to the Ti-27at\%V alloy, both electron diffraction patterns and dark-field images show that the DI- $\omega$ transformation hardly proceeds at $373 \mathrm{~K}$ as is seen from Figs. 3(c) and 3(f). Nevertheless, the diffuse scattering around the $\omega$-phase spots is strengthened after aging at $373 \mathrm{~K}$ although the diffraction spots hardly appear in electron diffraction patterns shown in Fig. 3(f). This means that even in the Ti-27at\%V alloy, which shows no $T_{\omega}$ or whose $T_{\omega}$ is far below RT, the phase fraction of the diffuse $\omega$ structure slightly increases upon aging at $373 \mathrm{~K}$, but a long-range order of the $\omega$ phase does not form. This fact is very interesting and is discussed later, together with the IXS measurement results.

\section{Hardness changes with DI- $\omega$ transformation}

Based on the fact that the DI- $\omega$ transformation can proceed at $373 \mathrm{~K}$, we here address the hardness changes with the proceeding of the DI- $\omega$ transformation at this temperature. Figure 4(a) shows the aging time dependence of the Vickers hardness normalized by the Vickers hardness measured immediately after the solid-solution treatment; the initial Vickers hardness values are shown in the table inset in Fig. 4(a). It can be seen from the table that the absolute value of Vickers 

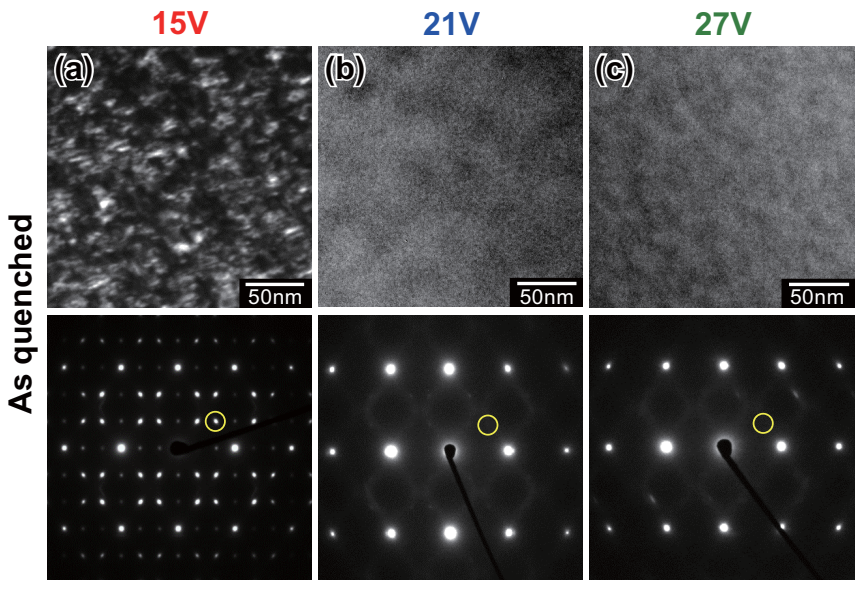

(g) $\overline{2} 2$

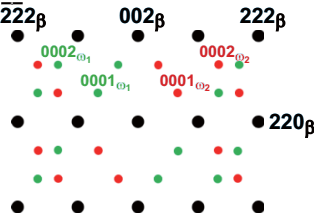

$15 \mathrm{~V}$

$21 \mathrm{~V}$
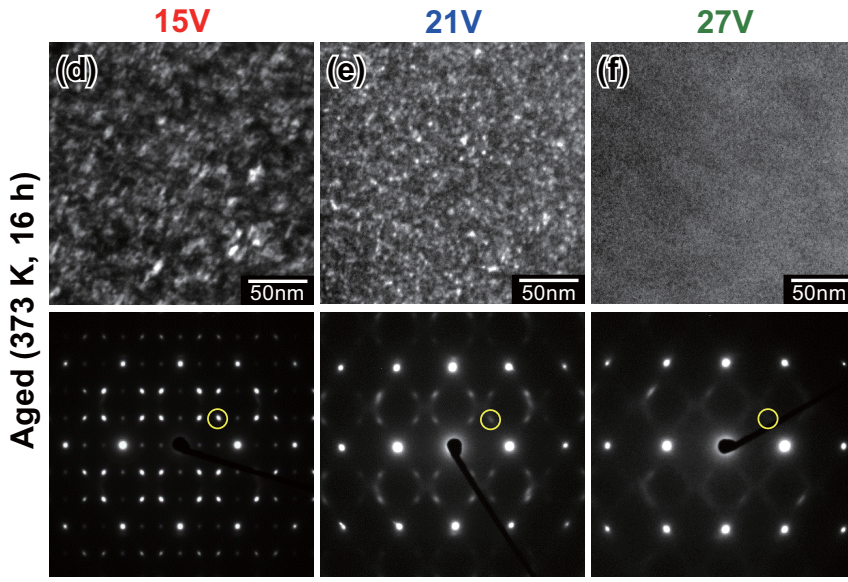

FIG. 3. Transmission electron microscope (TEM) observations for (a) Ti-15at $\% \mathrm{~V}$, (b) Ti-21at $\% \mathrm{~V}$, and (c) Ti-27at $\% \mathrm{~V}$ quenched from the solid-solution heat treatments, and for (d) Ti-15at\%V, (e) Ti$21 \mathrm{at} \% \mathrm{~V}$, and (f) $\mathrm{Ti}-27 \mathrm{at} \% \mathrm{~V}$ annealed at $373 \mathrm{~K}$ for $72 \mathrm{~h}$. Each upper picture is the dark field image generated by using the diffraction spot that is circled yellow in the lower electron diffraction pattern. $(\mathrm{g})$ Schematic figure showing electron diffraction spots coming from the $\beta$ and $\omega$ phases.

hardness of Ti-15at\% $\mathrm{V}$ is significantly higher than those of Ti-21at $\% \mathrm{~V}$ and Ti-27at\%V. This is because the athermal $\omega$ transformation occurs in Ti-15at\% V during quenching, which is consistent with the TEM observation.

It is clearly seen that the values of Vickers hardness increase with aging at $373 \mathrm{~K}$ for all the samples. The increase in Vickers hardness due to aging at $373 \mathrm{~K}$ where no diffusion occurs [as shown in Fig. 2(c)] is considered to be the same phenomenon as the RT-aging effect [31]. Although the Ti$15 \mathrm{at} \% \mathrm{~V}$ alloy undergoes the athermal $\omega$ transformation during the quenching treatment (as shown in Fig. 3), the increase ratio of the hardness is larger as the V composition is lower; the Vickers hardness increases after aging for $472 \mathrm{~h}$ by about $20 \%$ for $\mathrm{Ti}-15 \mathrm{at} \% \mathrm{~V}$, about $7 \%$ for $\mathrm{Ti}-21 \mathrm{at} \% \mathrm{~V}$, and about $1 \%$ for

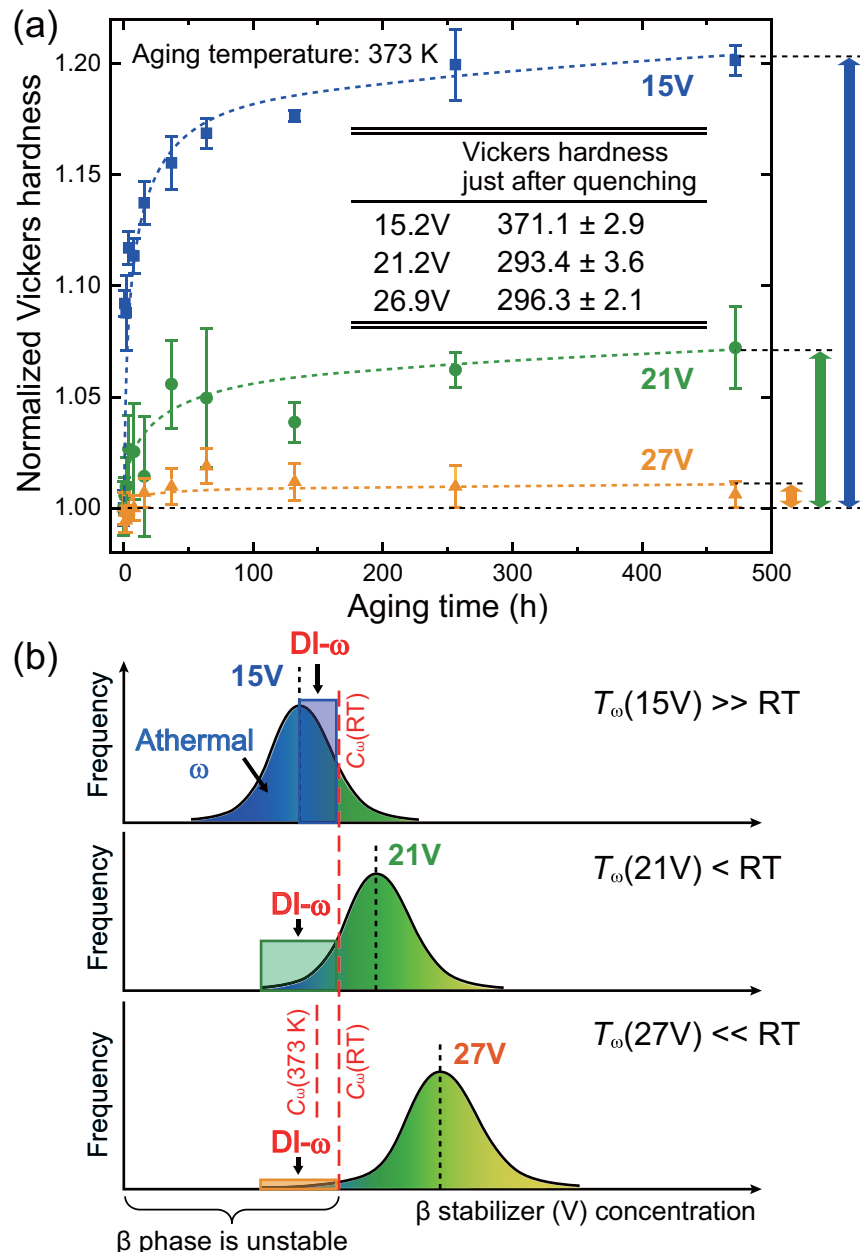

FIG. 4. (a) Change in Vickers hardness as a function of annealing time at $373 \mathrm{~K}$. The Vickers hardness is normalized by that of the as-quenched sample. (b) Interpretation of the hardness increase based on the concentration distribution of the $\beta$-stabilizing element (V) in reference to Fig. 1. The value of $C_{\omega}(\mathrm{RT})$ (red dashed line) represents the concentration at which the alloy undergoes the athermal transformation at $T_{\omega}=\mathrm{RT}$. When the median in the concentration distribution equals $C_{\omega}(\mathrm{RT})$, the alloy's $T_{\omega}$ is RT, therefore, when the median is located at a higher concentration than $C_{\omega}(\mathrm{RT})$, the alloy's $T_{\omega}$ is lower than RT. Each rectangle indicates the region that can undergo the DI- $\omega$ transformation at RT, and such a region would be a little bit narrower at $373 \mathrm{~K}$ because $C_{\omega}(373 \mathrm{~K})$ is shifted toward the left side.

Ti-27at\%V. Thus, it deserves notice that the Vickers hardness increases even after the occurrence of the athermal $\omega$ transformation (in Ti-15at\% V).

To understand this intriguing phenomenon, we try to grasp the mechanism of the hardness increase after aging at $373 \mathrm{~K}$ (which is slightly higher than RT but sufficiently low to prevent substantial atomic diffusion of $\mathrm{Ti}$ and V). Figure 4(b) shows the schematic illustration for the concentration distribution of the $\beta$-stabilizing (V) element. The red dashed line indicates the $\mathrm{V}$ composition $C_{\omega}(\mathrm{RT})$, the alloy of which exhibits $T_{\omega}=$ RT. Since the Ti-15at $\% \mathrm{~V}$ alloy exhibits $T_{\omega}$ higher than RT, the median of its concentration distribution is lower than $C_{\omega}(\mathrm{RT})$, whereas the median values for the other alloys 
(21 and 27at $\% \mathrm{~V})$ are surely higher than $C_{\omega}(\mathrm{RT})$ [therefore, these medians must be higher than $\left.C_{\omega}(373 \mathrm{~K})\right]$. Although $C_{\omega}(\mathrm{RT})$ is actually higher than $C_{\omega}(373 \mathrm{~K})$ since $C_{\omega}(\mathrm{RT})$ is close to $C_{\omega}(373 \mathrm{~K})$ according to the literature [35], in what follows, we simply use $C_{\omega}$ for these compositions.

As illustrated in Fig. 1, the V-poor local regions in the $\beta$ matrix can transform to the $\omega$ phase, that is, such regions having compositions below $C_{\omega}$ would have a possibility to undergo the athermal transformation during quenching. Most of the $\beta$-phase matrix that satisfies the above compositional condition is considered to undergo the transformation, but there would exist some untransformed regions not only above but also even below $C_{\omega}$. We claim that such regions can play a significant role in the DI- $\omega$ transformation. The large increase in hardness of Ti-15at\% V shown in Fig. 4(a) can be well interpreted as the above explanation (a large rectangular region means a large volume fraction). Even though the athermal transformation already occurs in this alloy, the DI- $\omega$ can proceed with low-temperature aging, so that the hardness gradually increases to a significant extent. In the case of Ti-21at $\% \mathrm{~V}$, although there exist some regions that can transform to $\omega$ since the median is higher than $C_{\omega}$, the athermal transformation hardly occurs as a whole. However, the quenched $\beta$ matrix in the Ti-21at\% V sample has the potential to undergo the $\omega$ transformation; therefore, the DI- $\omega$ mode can be observed. In contrast, there are very few regions below $C_{\omega}$ in Ti-27at\% V. Based on a statistical interpretation, we can successfully understand the change in the mechanical properties when the quenched Ti-V alloys with different $\mathrm{V}$ compositions are aged at low temperatures.

\section{Mechanical relaxation in the submegahertz frequency range}

The elementary process of the $\omega$ transformation, i.e., the dynamic $\{111\}_{\beta}$ collapse, can be detected as a mechanical relaxation by the internal friction (IF) measurement at low temperatures. Since the activation energy of the $\{111\}_{\beta}$ collapse is shown to be about $220 \mathrm{meV}$ [40] or $140-180 \mathrm{meV}$ [31], the IF measurement in the submegahertz frequency range must be conducted at low temperatures. Here, the present measurement aims to investigate whether the internal friction is indeed attributed to the dynamic $\{111\}_{\beta}$ collapse or not. Figure 5(a) shows the change in the resonance frequency $f_{\mathrm{r}}$ (of the sample) and internal friction $Q^{-1}$ in the Ti-21at\% V alloy during the heating process after quenching from $1073 \mathrm{~K}$ and during the cooling/heating processes after aging at $373 \mathrm{~K}$ for $72 \mathrm{~h}$. Since the elastic constants are approximately proportional to $f_{\mathrm{r}}^{2}$, they increase with decreasing temperature below $300 \mathrm{~K}$ (but this behavior depends on the kind of the elastic constants). It deserves to note that the inflection point ( $\Delta M$ effect) clearly appears around $180 \mathrm{~K}$, which does not follow the well-established Varshni equation [41]. This is because there exists a certain kind of mechanical relaxation responding to the periodic strain in the submegahertz frequency range.

The activation energy $\Delta E_{\{111\}}$ of the dynamic $\{111\}_{\beta}$ collapse is evaluated by analyzing the $Q^{-1}(T)$ curve of the Ti-21at\%V alloy using the Debye function

$$
Q^{-1}=\Delta \frac{\omega_{\mathrm{r}} \tau_{0} \exp \left(\Delta E_{\{111\}} / k T\right)}{1+\left[\omega_{\mathrm{r}} \tau_{0} \exp \left(\Delta E_{\{111\}} / k T\right)\right]^{2}},
$$
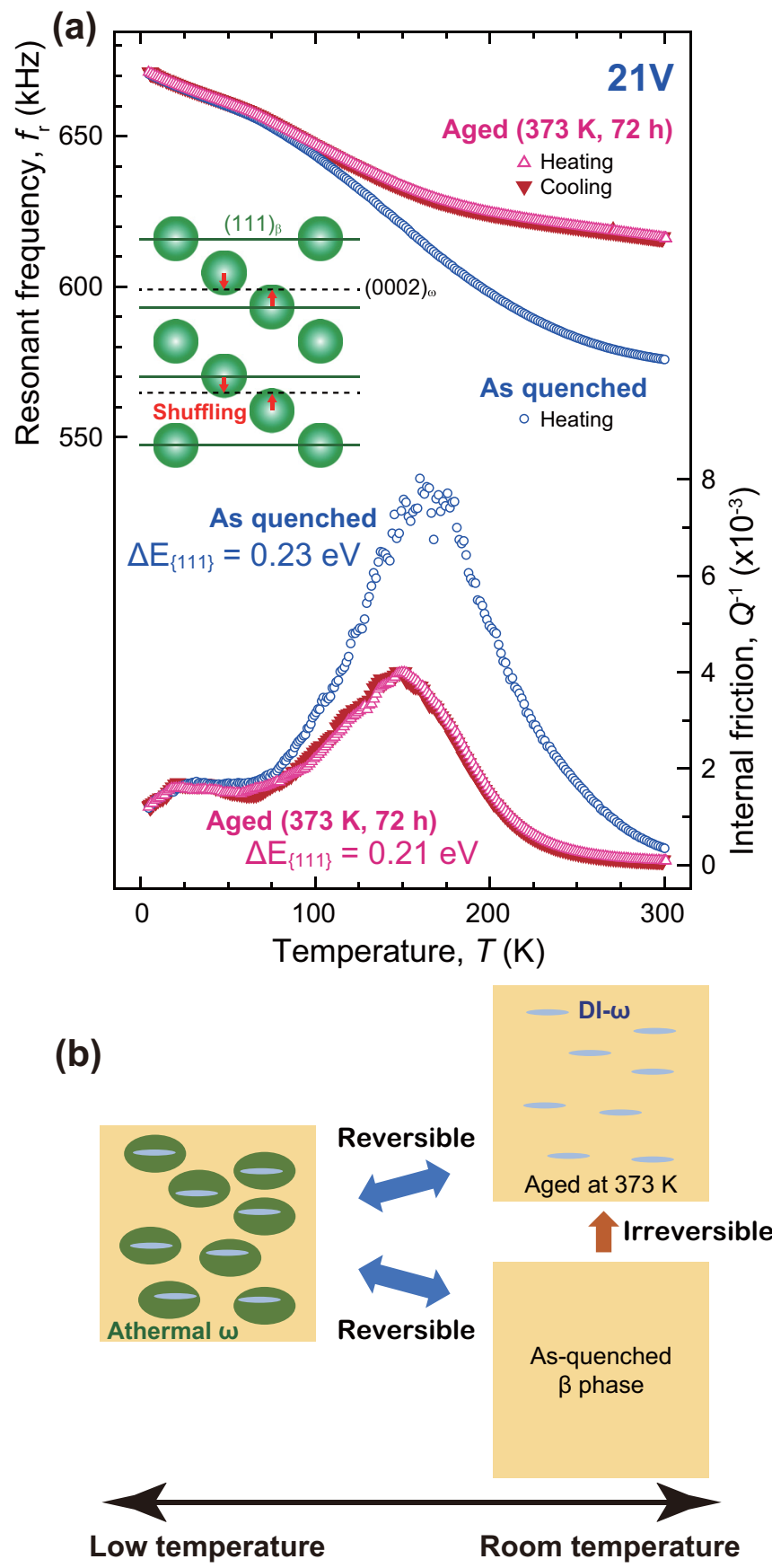

FIG. 5. (a) Resonance frequency and internal friction as a function of temperature in the submegahertz frequency range. The intensity of the internal friction (IF) after annealing at $373 \mathrm{~K}$ drastically decreases compared to that before annealing, substantiating that the IF peak is attributed to the activation process of the dynamic $\omega$ transformation. (b) Schematic illustration of the nucleation and growth of the athermal $\omega$ phase stemming from the DI- $\omega$ particles.

where $\Delta$ is the relaxation strength, $\omega_{\mathrm{r}}\left(=2 \pi f_{\mathrm{r}}\right)$ the angular frequency, and $\tau_{0}$ the time constant according to literature [40], $\tau_{0}=2.2 \times 10^{-14} \mathrm{~s}$. As indicated by our previous paper [31], such a broad IF profile cannot be correctly reproduced by the Debye function. Therefore, under the condition that $\omega_{\mathrm{r}} \tau=\omega_{\mathrm{r}} \tau_{0} \exp \left(\Delta E_{\{111\}} / k T_{\mathrm{p}}\right)=1$ holds at the peak position, we deduce only the activation energy $\Delta E_{\{111\}}$ by using the 
following equation:

$$
\Delta E_{\{111\}}=-k T_{\mathrm{p}} \ln \left(\omega_{\mathrm{r}} \tau_{0}\right) .
$$

From this peak analysis, we obtained the activation energy $\Delta E_{\{111\}}$ as small as $0.21-0.23 \mathrm{eV}$ for both as-quenched and aged samples, being in substantial agreement with the previous reports $[31,40]$. Although the activation energies for the two samples are almost unchanged, the IF peak intensity decreases drastically after aging at $373 \mathrm{~K}$ for $72 \mathrm{~h}$ (as seen from Figs. 3 and 4, quite some amount of the DI- $\omega$ phase is considered to be already formed after this aging). This indicates that the elementary process of the $\omega$ transformation is closely related to the IF peak, that is, the regions at which the DI- $\omega$ transformation has already been completed cannot contribute to the internal friction. Taking into account the previous studies $[40,42,43]$, it is concluded that the present IF peak is attributed to the elementary process, that is, the "dynamic $\{111\}_{\beta}$ collapse" of the $\omega$ transformation.

Furthermore, it is interesting to note that the two curves of $f_{\mathrm{r}}$ before and after aging merge into the same values at low temperatures; note that the same sample was used for the two measurements. Figure 5(b) illustrates its mechanism, in which the blue and green regions represent the DI- $\omega$ and athermal $\omega$ phases, respectively, in the $\beta$ phase matrix (yellow). The athermal $\omega$ phase can be formed during the cooling process from the DI- $\omega$ regions, and reversely transform to the $\beta$ phase during the heating process, as inferred from the virtually equal temperature dependence of the resonant frequency and internal friction for the cooling and heating processes after aging [Fig. 5(a)]. This is a very important implication in that the athermal $\omega$ transformation stems from a local unstable $\beta$ region at which the DI- $\omega$ mode can proceed above $T_{\omega}$. It is considered that the reason why the DI- $\omega$ phase cannot reversely transform to the $\beta$ phase around $\mathrm{RT} \sim 373 \mathrm{~K}$ would be that a certain stabilization/modification of the $\omega / \beta$ interface occurs with some defects. In the case of the Ti-27at $\% \mathrm{~V}$ alloy, as shown in the Supplementary Information of our previous paper [31], the IF peak can be observed in a similar temperature range, but unlike $\mathrm{Ti}-21 \mathrm{at} \% \mathrm{~V}$, the resonant frequencies of as-quenched and postaged samples do not merge into one curve for Ti-27at $\% \mathrm{~V}$ because the alloy does not exhibit an obvious athermal $\omega$ transformation at any temperature.

\section{E. Inelastic x-ray scattering/ultrasound measurements for Ti-27at $\% \mathrm{~V}$ alloy showing no athermal $\omega$ transformation}

In the Ti-27at\% V alloy, in which the athermal $\omega$ transformation is not expected to occur, no microstructural effect is additionally influencing the temperature dependence of the elasticity. Therefore, it should be possible to investigate the elementary process of the $\omega$ transformation within the Ti$27 \mathrm{at} \% \mathrm{~V}$ alloy. We investigate whether or not a certain kind of relaxation can be observed in the terahertz frequency range by using the IXS measurement and compared the results to the submegahertz ultrasound (US) measurement. Specifically, we focus on how the [111] longitudinal elastic constant as a function of temperature differs depending on the measurement frequency range.

Dynamic structure factors $S(\vec{Q}+d \vec{Q}, \omega)$ at $\vec{Q}=(2,2,2)$ and $d \vec{Q}=(-0.05,-0.05,-0.05)$ measured at 80 and $150 \mathrm{~K}$ are shown in Fig. 6(a); as clearly seen by the red dashed line, the excitation energy measured at $80 \mathrm{~K}$ is larger than that at $150 \mathrm{~K}$. To obtain the excitation energy at each $\vec{Q}$ position, the present IXS data were fitted to the damped harmonic oscillator model [44]

$$
\frac{S(\vec{Q}, \omega)}{S(\vec{Q})}=A_{0} \frac{\Gamma_{0}}{\Gamma_{0}^{2}+\omega^{2}}+A_{Q} \frac{\Omega_{Q}^{2} \Gamma_{Q}}{\left(\omega^{2}-\Omega_{Q}^{2}\right)^{2}+\omega^{2} \Gamma_{Q}^{2}},
$$

where $A_{0}$ and $A_{Q}$ are the relative intensities, $\Gamma_{0}$ and $\Gamma_{Q}$ are associated with the sound attenuation, and $\Omega_{Q}$ is the characteristic acoustic frequency. In practice, we included the detailed-balance term and convolved it in the instrumental resolution function. The sound velocity of the longitudinal wave along the $[111]_{\beta}$ axis, $V_{[111]}=\Omega_{Q} /|\vec{Q}|$, was obtained by linearly fitting the excitation energy values at the two opposite arm positions: $d \vec{Q}=( \pm 0.05, \pm 0.05, \pm 0.05)$. The longitudinal elastic constant along the $[111]_{\beta}$ axis was derived by $C_{[111]}=\rho V_{[111]}^{2}$ with the sound velocity and mass density $\left(\rho=4846.5 \mathrm{~kg} / \mathrm{m}^{3}\right)$.

Figure 6(b) compares the longitudinal elastic constants along the $[111]_{\beta}$ axis obtained from the IXS and US measurements. Both the IXS and US elasticity decreases with decreasing temperature until around $100-150 \mathrm{~K}$, being a reversal of the common trend of crystal lattices, which indicates that the unstable $\beta$ matrix tries to undergo the $\omega$ transformation even though it has no $T_{\omega}$. Below around $100-150 \mathrm{~K}$, both the IXS and US elasticity tends to increase with decreasing temperature. It should be noted that the IXS elasticity steeply increases with decreasing temperature whereas the US elasticity increases moderately.

First, we discuss the origin of the increase in the elasticity with decreasing temperature. There are two possible reasons for the stiffening: (i) the diminishing of the anharmonic effect due to the decreased contribution of the higher-order terms of the potential at low temperatures as is usually observed in conventional crystal lattices (hereafter, called the "inverse anharmonic effect") and (ii) the so-called $\Delta M$ effect that emerges when the external frequency becomes high compared to that of the relaxation process (the elementary dynamics of the $\beta \leftrightarrow \omega$ transformation become frozen). Figure 6(c) shows the characteristic frequency $(f=1 / \tau)$ for the relaxation process with representative activation energies of $0.1,0.2$, and $0.3 \mathrm{eV}$ as a function of temperature. In reality, the activation energy will have a wider variation due to the compositional fluctuation at the nanometer scale. As seen in Fig. 6(c), the $\mathrm{THz}$ (IXS) frequency is higher than the characteristic frequency over the whole temperature range investigated. Thus, the IXS measurement will yield the instantaneous elasticity, which is independent of external frequencies and, hence, the substance-specific temperature dependence of elasticity becomes available. Therefore, the $\Delta M$ effect cannot rationalize the temperature dependence of the IXS elasticity, and the inverse anharmonic effect can exclusively be attributed to the stiffening with decreasing temperature. On the other hand, since the $\mathrm{MHz}$ (ultrasound) frequency crosses the characteristic frequency band, the US measurement yields a transient elasticity from relaxed to unrelaxed upon cooling. Taking into consideration the compositional fluctuation, the US waves will still experience local soft regions (relaxed) at low 

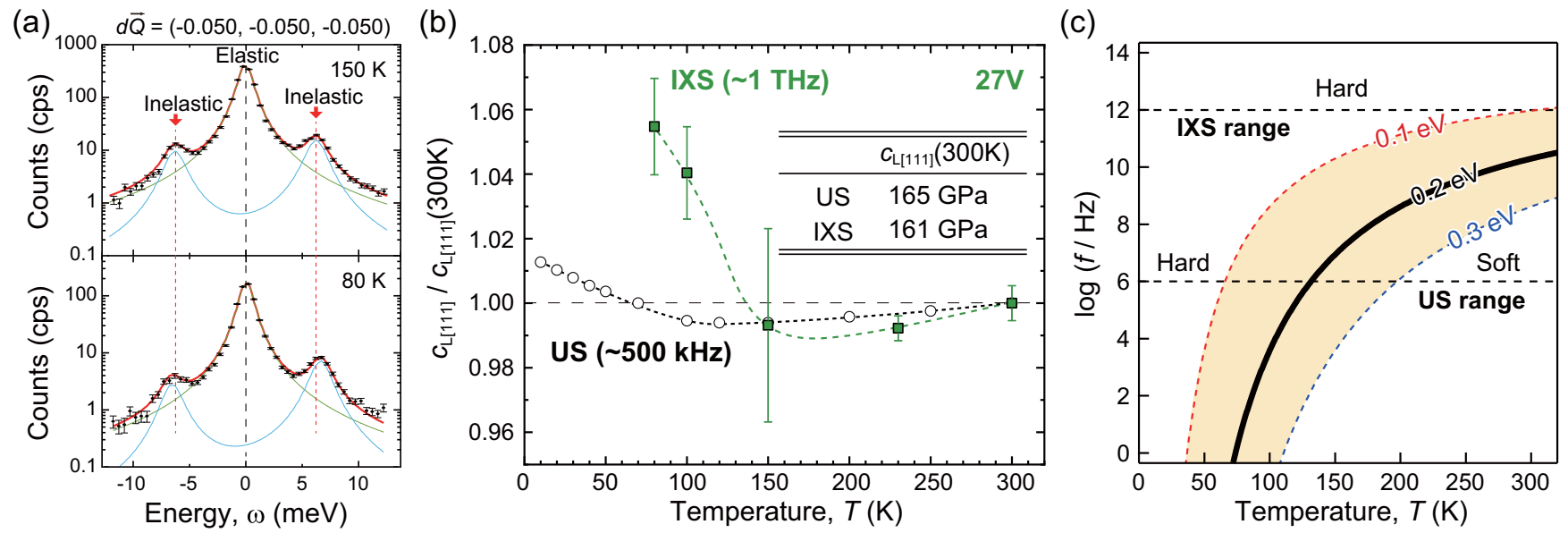

FIG. 6. Inelastic x-ray scattering (IXS) measurement for Ti-27at $\% \mathrm{~V}$ at low temperatures. (a) The dynamic structure factors obtained at 150 and $80 \mathrm{~K}$. (b) The longitudinal elastic constant along the $\{111\}_{\beta}$ axis. The inset shows the absolute values at $300 \mathrm{~K}$. (c) The logarithm of the characteristic frequency of the elementary process of the $\{111\}_{\beta}$ collapse versus temperature for activations with $\Delta E=0.1,0.2$ (average), and $0.3 \mathrm{eV}$ and $\tau_{0}=2.2 \times 10^{-14} \mathrm{~s}$. The phonon frequency measured by the IXS method is always higher than the characteristic frequency in the whole temperature range below $300 \mathrm{~K}$, whereas the frequency measured by the ultrasound (US) method crosses the characteristic frequency band with the temperature variation.

temperatures. Therefore, the increase in the US elasticity with decreasing temperature is partly due to the $\Delta M$ effect, and also partly due to the inverse anharmonic effect. Nevertheless, it seems curious that only marginal increase in the US elasticity is experimentally observed despite the dual stiffening effects.

Then, we discuss why the stiffening in the US elasticity appears to such a slight degree in terms of the concept of elastic inhomogeneity, which one of the authors advocated to comprehend the relationship between elasticity and fragility in metallic glasses [45]. Within the $\beta$ matrix, some local regions, which attempt to transform to $\omega$, remain soft due to the instability of the $\beta$ lattice while other local regions of less instability, which cannot undergo the elementary $\beta \leftrightarrow \omega$ motion, become stiffer due to the inverse anharmonic effect. Thus, there exists an elastic inhomogeneity at the nanometer scale at low temperatures due to the concentration fluctuation with keeping the lattice coherence (i.e., full lattice continuity). In view of the wavelength, the coherence lengths for the IXS and US measurements will be a few $\mathrm{nm}$ and $\mathrm{mm}$, respectively. This difference in the coherence length would render the perceptibility for the local stiffening in different ways; the US waves preferentially experience the softer regions considering a series model of the elastic continuum, resulting in the less pronounced increase in the elastic constant at low temperatures, whereas the IXS efficiently detects the local stiffening due to the inverse anharmonic effect at low temperatures.

\section{WHAT IS THE DIFFERENCE BETWEEN THE WELL-ESTABLISHED $\omega$ MODES AND DIFFUSIONLESS ISOTHERMAL $\omega$ MODE?}

First, we discuss the difference between the conventional isothermal $\omega$ mode and the DI- $\omega$ mode. The conventional isothermal $\omega$ mode occurs via diffusion-mediated phase separation by which solute-lean regions are produced. During the phase separation process, the solute concentration of the solute-lean regions becomes lower than $C_{\omega}$ so that the omega phase is formed in these regions. The conventional isothermal $\omega$ mode exhibits an incubation period due to the diffusionmediated phase separation. In contrast to the conventional isothermal $\omega$ mode, no diffusion process is required for the DI- $\omega$ mode. In the DI- $\omega$ mode, local solute-lean $\beta$ regions that inevitably exist for statistical reasons are unstable and can transform into a temporary $\omega$ structure even if the whole $\beta$ matrix is stable on average. The DI- $\omega$ mode also exhibits an incubation period because it requires some sort of nucleation process in order to form a permanent $\omega$ phase.

Second, we discuss the difference between the athermal $\omega$ and DI- $\omega$ modes. In our opinion, the most crucial difference between them is whether the $\omega$ transformation occurs at the median or tail of the concentration distribution of the $\beta$-stabilizing element in Fig. 1(b). The athermal $\omega$ transformation occurs when the temperature goes below $T_{\omega}$. Then, what determines $T_{\omega}$ ? This is an important question for understanding the fundamentals of $\omega$ transformation in $\beta$-Ti alloys. The athermal transformation usually occurs reversibly around $T_{\omega}$ with little temperature hysteresis. These characteristics look like a second-order transition, but the transition proceeds via a nucleation and growth process. Therefore, $T_{\omega}$ is interpreted as a temperature where most (e.g., half) of the $\beta$ matrix (regions below the median in the concentration distribution) can begin to transform to the $\omega$ phase. In the present concept, the threshold value $50 \%$ is used for the definition of $T_{\omega}$.

The key to understanding these peculiar features of the $\omega$ transformation could be a temperature dependence of the interfacial energy between the $\omega$ and $\beta$ phases. As suggested previously in the paper by Tane et al. [31], the nucleation of the $\omega$ phase is controlled by this interfacial energy. As pointed out here, the elementary motion takes place with a very small activation energy of the order $100-200 \mathrm{meV}$, so this process cannot substantially be a controlling factor of the $\omega$ transformation even at low temperatures. Therefore, the crucial factor controlling the phenomenon is assumed to be the 
nucleation activation energy $\Delta W^{*}$. It should be noted here that the activation energy for the $\omega$-nucleation is fundamentally different from that of the $\{111\}_{\beta}$ collapse. In both the diffusionless isothermal and the athermal $\omega$ transformations, the transformation rate is dominated by the product of the probabilities of two activation processes: a dynamic collapse of $\{111\}_{\beta}$ pairs forming a temporary $\omega$ structure (as discussed in the present work), and a nucleation process forming a permanent $\omega$ phase [31]. As mentioned above, the first activation energy is of the order 100-200 meV, so that the dynamic collapse of $\{111\}_{\beta}$ readily occurs even at RT. In the nucleation process, the interfacial energy $\gamma$ between the $\beta$ and $\omega$ phases plays a crucial role to dominate the nucleation rate because the nucleation barrier $\Delta W^{*}$ is given by $\Delta W^{*} \sim \gamma^{3} / \Delta \mu^{2}$, where $\Delta \mu$ is the free energy difference between the $\omega$ and $\beta$ phases. Since the elasticity of the $\beta$ phase is significantly softened around $T_{\omega}$ [7], the nucleation of the $\omega$ phase in the athermal transformation becomes much easier. In contrast, the nucleation in solute-lean local regions in the DI- $\omega$ transformation requires relatively high activation energy since most of the $\beta$ regions are still thermodynamically and elastically stable. The kinetics of the DI- $\omega$ transformation is governed mainly by such a high activation energy of the nucleation process. Therefore, although the elemental process of $\omega$ transformation itself can frequently occur above around $150 \mathrm{~K}$, the DSC peak corresponding to the DI- $\omega$ starts to appear only above $350-400 \mathrm{~K}$ at the fast scan speed of $10 \mathrm{~K} / \mathrm{min}$ as shown in Fig. 2(a). On the other hand, the conventional isothermal $\omega$ transformation begins to occur at around $573 \mathrm{~K}$, which obscures the tail of the DI- $\omega$ peak at the high-temperature side. Thus, the peak width of the DI- $\omega$ transformation appears relatively narrow in the DSC curves.

\section{CONCLUSION}

We explored various characteristic features of the diffusionless isothermal $\omega$ transformation (DI- $\omega$ ) based on the mechanism proposed in our previous paper [31]. Through this work, we reached the following conclusions.

(1) The DI- $\omega$ transformation can be detected by a DSC measurement, which shows that the DI- $\omega$ transformation is an exothermic reaction, at temperatures where substantial atomic diffusion cannot occur.
(2) The mechanical hardening due to the DI- $\omega$ transformation can be interpreted by the concept of the concentration distribution of the $\beta$-stabilizing element.

(3) When the median value in the concentration distribution crosses the $T_{\omega}$ curve, which is the athermal $\omega$ transformation temperature as a function of the macroscopically averaged concentration, most of the matrix can undergo an athermal $\omega$ transformation.

(4) The present submegahertz internal-friction measurements show that the elasticity of the aged sample equals that of the quenched sample at low temperatures, even though the values of the former are obviously higher than those of the latter at room temperature. This suggests that the athermal $\omega$ is likely formed around the regions where the DI- $\omega$ can proceed even above the athermal $\omega$ transformation temperature $T_{\omega}$.

(5) By comparing the IXS and US elasticity, it is evident that the elastic softening occurs even for the $\beta$-stabilized Ti alloys (for example, in the Ti-27at\%V alloy). After completely freezing the elementary motion of the $\{111\}_{\beta}$ collapse, an elastic stiffening occurs due to the inverse anharmonic effect with decreasing temperature, which is more effectively detected by the IXS measurement.

(6) The difference between the athermal and diffusionless isothermal modes manifests itself in the elastic softening of the $\beta$ matrix. Namely, when the matrix elasticity has softened enough at a low temperature (that is equal to $T_{\omega}$ ), the athermal $\omega$ transformation can occur easily in most of the $\beta$ matrix, whereas the DI- $\omega$ mode gradually proceeds via a nucleation process with a relatively high $\omega / \beta$ interfacial energy.

\section{ACKNOWLEDGMENTS}

This work was supported by JST, PRESTO Grant No. JPMJPR1996, and JSPS KAKENHI Grant No. 19H04408, Japan. Support by the GIMRT Program of the Institute for Materials Research, Tohoku University (Grant No. 19K0513) is gratefully acknowledged. The synchrotron radiation experiment was performed at the BL35XU of SPring-8 with the approval of the Japan Synchrotron Radiation Research Institute (JASRI) (Proposal No. 2018B1047).
[1] T. Saito, T. Furuta, J. H. Hwang, S. Kuramoto, K. Nishino, N. Suzuki, R. Chen, A. Yamada, K. Ito, Y. Seno, T. Nonaka, H. Ikehata, N. Nagasako, C. Iwamoto, Y. Ikuhara, and T. Sakuma, Science 300, 464 (2003).

[2] D. Banerjee and J. C. Williams, Acta Mater. 61, 844 (2013).

[3] M. Long and H. J. Rack, Biomaterials 19, 1621 (1998).

[4] H. Y. Kim, Y. Ikehara, J. I. Kim, H. Hosoda, and S. Miyazaki, Acta Mater. 54, 2419 (2006).

[5] A. Devaraj, V. V. Joshi, A. Srivastava, S. Manandhar, V. Moxson, V. A. Duz, and C. Lavender, Nat. Commun. 7, 11176 (2016).

[6] M. Bönisch, A. Panigrahi, M. Stoica, M. Calin, E. Ahrens, M. Zehetbauer, W. Skrotzki, and J. Eckert, Nat. Commun. 8, 1429 (2017).
[7] M. Tane, T. Nakano, S. Kuramoto, M. Hara, M. Niinomi, N. Takesue, T. Yano, and H. Nakajima, Acta Mater. 59, 6975 (2011).

[8] P. D. Frost, W. M. Parris, L. L. Hirsch, J. R. Doig, and C. M. Schwartz, Trans. Amer. Soc. Metals 46, 231 (1954).

[9] B. S. Hickman, J. Mater. Sci. 4, 554 (1969).

[10] D. de Fontaine, N. E. Paton, and J. C. Williams, Acta Metall. 19, 1153 (1971).

[11] S. K. Sikka, Y. K. Vohra, and R. Chidambaram, Prog. Mater. Sci. 27, 245 (1982).

[12] S. Nag, A. Devaraj, R. Srinivasan, R. E. A. Williams, N. Gupta, G. B. Viswanathan, J. S. Tiley, S. Banerjee, S. G. Srinivasan, H. L. Fraser, and R. Banerjee, Phys. Rev. Lett. 106, 245701 (2011). 
[13] M. J. Lai, C. C. Tasan, J. Zhang, B. Grabowski, L. F. Huang, and D. Raabe, Acta Mater. 92, 55 (2015).

[14] D. Choudhuri, Y. Zheng, T. Alam, R. Shi, M. Hendrickson, S. Banerjee, Y. Wang, S. G. Srinivasan, H. Fraser, and R. Banerjee, Acta Mater. 130, 215 (2017).

[15] J. L. Zhu, S. Cao, Y. Wang, R. Yang, and Q. M. Hu, Comput. Mater. Sci. 155, 524 (2018).

[16] J. Y. Yan and G. B. Olson, J. Alloys Compd. 673, 441 (2016).

[17] B. Hu, S. Sridar, L. Hao, and W. Xiong, Intermetallics 122, 106791 (2020).

[18] E. L. Pang, E. J. Pickering, S. I. Baik, D. N. Seidman, and N. G. Jones, Acta Mater. 153, 62 (2018).

[19] Y. Zheng, R. E. A. Williams, D. Wang, R. Shi, S. Nag, P. Kami, J. M. Sosa, R. Banerjee, Y. Wang, and H. L. Fraser, Acta Mater. 103, 850 (2016).

[20] A. Planes, E. Obradó, A. Gonzalez-Comas, and L. Mañosa, Phys. Rev. Lett. 79, 3926 (1997).

[21] S. Singh, B. Dutta, S. W. D'souza, M. G. Zavareh, P. Devi, A. S. Gibbs, T. Hickel, S. Chadov, C. Felser, and D. Pandey, Nat. Commun. 8, 1006 (2017).

[22] S. M. Shapiro, Y. Noda, Y. Fujii, and Y. Yamada, Phys. Rev. B 30, 4314 (1984).

[23] S. Sarkar, X. B. Ren, and K. Otsuka, Phys. Rev. Lett. 95, 205702 (2005).

[24] D. Wang, Z. Zhang, J. Zhang, Y. M. Zhou, Y. Wang, X. D. Ding, Y. Z. Wang, and X. B. Ren, Acta Mater. 58, 6206 (2010).

[25] Y. C. Ji, D. Wang, X. D. Ding, K. Otsuka, and X. B. Ren, Phys. Rev. Lett. 114, 055701 (2015).

[26] D. Shindo, Y. Murakami, and T. Ohba, MRS Bull. 27, 121 (2002).

[27] C. B. Walker, Phys. Rev. B 28, 674 (1983).
[28] Z. Fan, Scr. Metall. Mater. 31, 1519 (1994).

[29] Y. Al-Zain, Y. Sato, H. Y. Kim, H. Hosoda, T. H. Nam, and S. Miyazaki, Acta Mater. 60, 2437 (2012).

[30] M. Tane, K. Hagihara, M. Ueda, T. Nakano, and Y. Okuda, Acta Mater. 102, 373 (2016).

[31] M. Tane, H. Nishiyama, A. Umeda, N. L. Okamoto, K. Inoue, M. Luckabauer, Y. Nagai, T. Sekino, T. Nakano, and T. Ichitsubo, Phys. Rev. Materials 3, 043604 (2019).

[32] L. D. Landau and E. M. Lifshitz, Statistical Physics: Vol. 5: Course of Theoretical Physics (Pergamon, Oxford, 1968).

[33] T. Ichitsubo and K. Tanaka, Phys. Rev. E 63, 060101(R) (2001).

[34] N. L. Okamoto, S. Kasatani, M. Luckabauer, M. Tane, and T. Ichitsubo, Scritpa Mater. 188, 88 (2020).

[35] N. E. Paton and J. C. Williams, Scr. Metall. 7, 647 (1973).

[36] M. Hirao and H. Ogi, EMATs for Science and Industry: Noncontacting Ultrasonic Measurements (Kluwer Academic, Dordrecht, The Netherlands, 2013).

[37] A. Q. R. Baron, Y. Tanaka, S. Goto, K. Takeshita, T. Matsushita, and T. Ishikawa, J. Phys. Chem. Solids 61, 461 (2000).

[38] J. F. Murdock and C. J. Mchargue, Acta Metall. 16, 493 (1968).

[39] G. Aurelio, A. F. Guillermet, G. J. Cuello, and J. Campo, Metall. Mater. Trans. A 33, 1307 (2002).

[40] A. W. Sommer, S. Motokura, K. Ono, and O. Buck, Acta Metall. 21, 489 (1973).

[41] Y. P. Varshni, Phys. Rev. B 2, 3952 (1970).

[42] C. W. Nelson, D. F. Gibbons, and R. F. Hehemann, J. Appl. Phys. 37, 4677 (1966).

[43] J. E. Doherty and D. F. Gibbons, Acta Metall. 19, 275 (1971).

[44] B. Fak and B. Dorner, Physica B 234, 1107 (1997).

[45] T. Ichitsubo, S. Hosokawa, K. Matsuda, E. Matsubara, N. Nishiyama, S. Tsutsui, and A. Q. R. Baron, Phys. Rev. B 76, 140201(R) (2007). 